\title{
A Case of Neurocysticercosis Clinically Mimicking Epilepsy: Report from Remote Hilly Area of Bangladesh
}

\author{
Suman Chowdhury ${ }^{1}$, Mohammad Najim $\operatorname{Uddin}^{2}$
}

${ }^{1}$ Resident Medical Officer, Rowangchhari Upazilla Health Complex, Bandarban Hilly District, Bangladesh; ${ }^{2}$ Assistant Professor (Neurology), Chittagong Ma-O-Shishu Medical College General Hospital, Chittagong, Bangladesh

[Received: 1 April 2016; Accepted: 15 May 2016; Published: 1 June 2016]

\begin{abstract}
Neurocysticercosis (NCC) is considered a common infection of the nervous system caused by the Taenia solium and is known to be the primary cause of preventable epilepsy in many developing countries. NCC is commonly resulted by the ingestion of Taenia solium eggs after consuming undercooked pork, or contaminated water. The parasite can grow in the brain and spinal cord within the nervous system, causing severe headache and seizures beside other pathological manifestations. People in North Eastern hilly areas of Bangladesh are very much fond of Pork meat. Here in this case report, an elderly indigenous male was found to be presented with weakness and sleep deprivation initially. During subsequent conversation with the patient for obtaining his medical history, he started complaining of dizziness, which eventually led to generalized convulsions lasting for about one minute. Though his neurological system examination was inconclusive, stool routine microscopic examination showed ova of $T$. solium. $\operatorname{IgG}$ antibody against $T$. solium was positive. Later, the patient was advised to have contrast MRI of brain which revealed multiple Ring enhancing T2 hyperintense cystic lesion with tiny central in different parts of the brain. [Bangladesh Journal of Infectious Diseases 2016;3(1):24-27]
\end{abstract}

Keywords: Indigenous; neurocysticercosis; Taenia solium; epilepsy; remote hilly area

Correspondence: Dr. Suman Chowdhury, Resident Medical Officer (RMO), Rowangchhari Upazilla Health Complex, Bandarban Hilly District, Chittagong, Bangladesh; Flat\# C-3, House\#74; Road\#06; O.R Nizam R/A, Chittagong, Bangladesh; Email: drsuman_chowdhury@yahoo.com; Cell no.: +8801712271839

How to cite this article: Chowdhury S, Uddin MN. Neurocysticercosis: A Case Report at Remote Hilly Area in Bangladesh. Bangladesh J Infect Dis 2016;3(1):24-27

Contributions to authors: Dr. Suman Chowdhury was involved in the diagnosis and management of the case. Dr. Mohammad Najim Uddin reviewed literature and drafted the manuscript.

Competing interests: We declare that we have no conflict of interest.

\section{Introduction}

Neurocysticercosis (NCC) is one of the seven neglected endemic zoonoses targeted by the World Health Organization ${ }^{1}$. It is considered a common infection of the nervous system and is known to be the primary cause of preventable epilepsy in many developing countries ${ }^{2-4}$. Epidemiologic studies have Bangladesh J Infect Dis demonstrated tight clustering household or household contacts with NCC patients have three times higher risk having positive serology for cysticercosis compared with controls ${ }^{5}$. NCC is a common cause of neurological disease transmitted through parasites called Taenia solium (T. solium) ${ }^{6}$. It is endemic in Africa, Asia and Latin America, making it the major cause of epilepsy and June 2016 | Volume 3 | Number 1 
convulsion in these regions ${ }^{7}$. It can be transmitted from human-to-human through a fecal-oral route. Contaminated food and water are the major sources of this infection ${ }^{8}$. The single cyst infection $(47.7 \%$ $53.4 \%)$, is the most common in Indian subcontinent ${ }^{9}$.

The age-adjusted prevalence of epileptic disease associated with NCC ranges from 10 to 15 per 1 000 inhabitants of tropical countries. Other manifestations of the disease include stroke, dementia, hydrocephalus and vision loss. It is estimated to cause 50,000 deaths every year ${ }^{7}$. This present case report was found in a remote area of Rowangcchari Upazilla in Bandarban Hilly district presenting with the clinical features of neurocysticercosis, especially epilepsy.

\section{Case Presentation}

This 55 years old Indigenous male (Figure I), non diabetic, normotensive, presented to the outpatient department in a Upazilla Health Complex, Rowangchhari, Bandarban Hilly District, with weakness and impaired sleep for few weeks. During the initial conversation with the patient for obtaining the medical history, the patient started complaining of dizziness, which eventually led to generalized convulsions that lasted for about one minute. After recovery the patient added that he has been taking an antiepileptic drug (AED) for last 2 years prescribed by a local physician. On further elaboration, the past attacks were focal epilepsy with secondary generalization. The patient also admitted to have headache in between attacks sometimes for last few years. Headache was of moderate intensity, involving both sides and back. There were no aggravating or relieving factors, no diurnal variation.

There was also no association with vomiting and blurring of vision. The patient did not mention any history of fever and weight loss. No history of same illness was provided among the rest of his family members. On examination, he was co operative and very much well behaved. There was mild anaemia, but no lymphadenopathy, clubbing, bodyache or skin rash. On Neurological examination, he was well oriented. GCS was normal (15/15). There were no features of focal weakness. Muscle bulk and tone was normal. Planter reflexes were bilaterally flexor. All the cranial nerves were intact, though mild impairment of sensory system was noticed on lower limbs regarding vibration. Gait was antalgic probably due to osteoarthritis of right knee. The patient did not have any significant cerebellar function abnormalities.

Bangladesh J Infect Dis

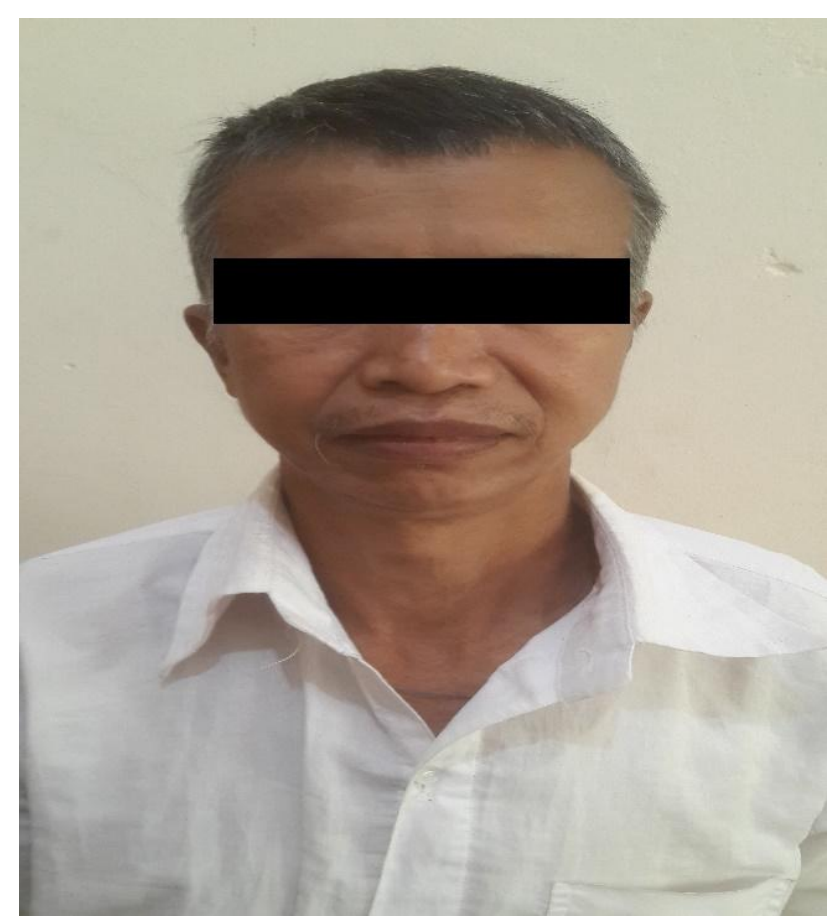

Figure I: The 55 years old indigenous man

There was no papillaedema on fundoscopic examination. As a habitant of the Hilly areas like Rowangchhari Upazilla under the district Bandarban, he along with other family members were very much used to take pig meat frequently, unlike any other parts of this populated country.

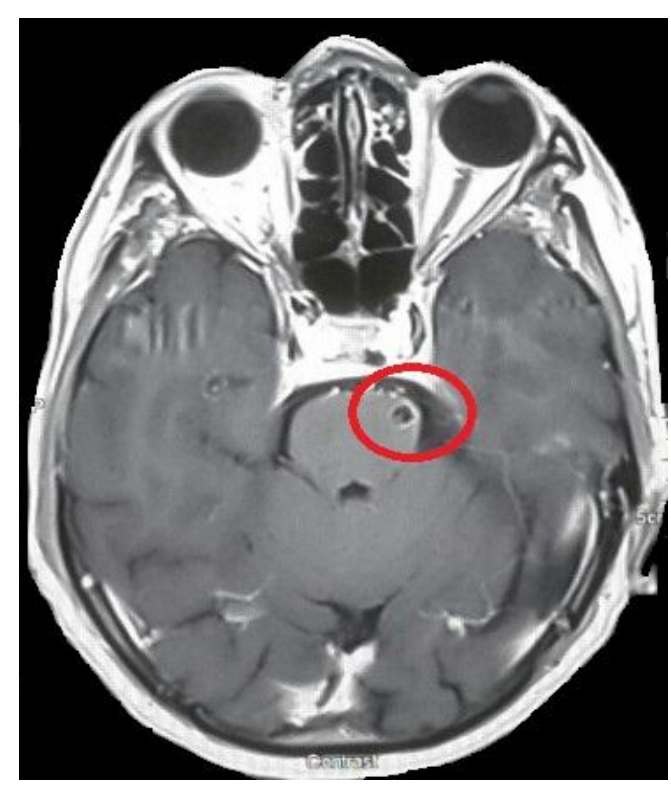

Figure II: Contrast MRI of Brain showing Ring enhancing T2 hyperintense cystic lesion with tiny central dot (red circle) in the Left pons

Moreover, due to lack of proper food hygiene and particular cooking technique, it was quite likely that they would have been infested with such helminthic diseases. In addition to these, lack of health 
education and hard to reach locality, they were not under the coverage of regular antihelminthic mediation, or irradiation campaign. Routine examination of blood revealed mild anaemia (9.2 $\mathrm{gm} / \mathrm{dl}$ ). Stool routine microscopic examination showed ova of $T$. solium. IgG antibody (ELISA test with RIDASCREEN ${ }^{\circledR}$ Taenia Solium IgG from RBiopharm AG, Germany) against $T$. solium was positive. Later, the patient was advised to have contrast MRI of brain which revealed multiple Ring enhancing T2 hyperintense cystic lesion with tiny central in the Left pons anterior aspect (Figure II), left cingulate gyri (Figure III), left post central gyri, right posterior parietal cortex, right temporal horn of lateral ventricle and right temporal cortex. Given the deep-seated mass, a biopsy or any surgical procedure was deferred. The patient is currently taking Carbamazepine $800 \mathrm{mg}$ per day and albendazole $400 \mathrm{mg}$ twice a day and he is now seizure free.

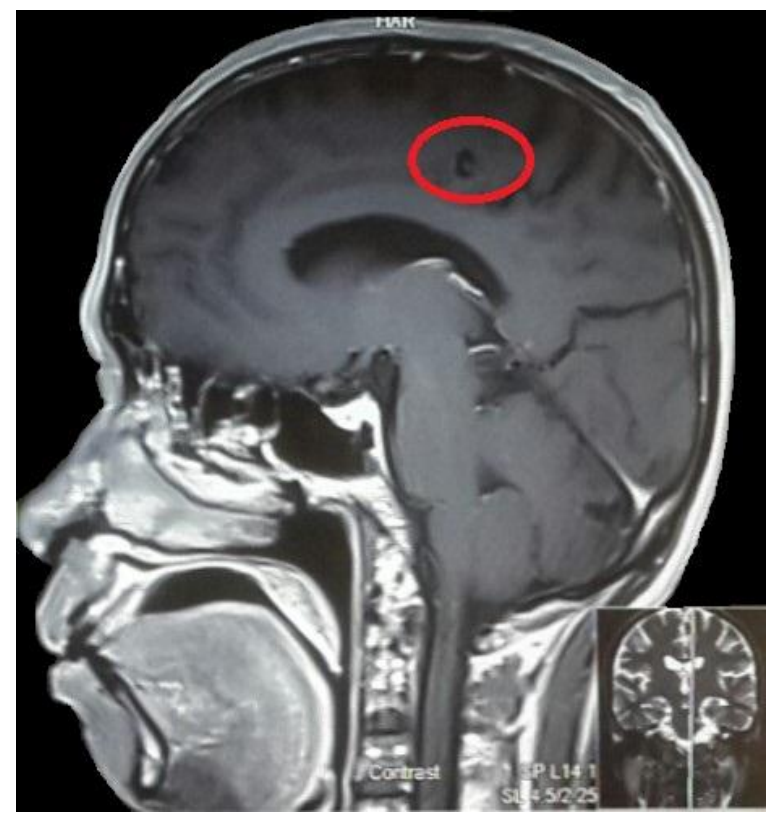

Figure III: Contrast MRI of Brain showing Ring enhancing T2 hyperintense cystic lesion with tiny central dot (red circle) in the Left cingulate gyri

\section{Discussion}

T. solium, commonly known as adult tape worm, is the parasite involved in pathogenesis of NCC. Human infected with adult tape worms can reside in the small intestine for years. The worms may develop up to 7 meter in length with each proglottid segment containing 50000 to 100000 eggs which eventually shed the eggs in feces, which can contaminate water or be ingested by an intermediate host, mostly pigs. The eggs hatch and release the larvae (cysticerci) into the intermediate host. The larvae penetrate the intestinal wall and spread through the blood stream to different organs in the body such as brain, striated muscles, liver and other tissues. The cycle is complete when human, the definitive host, ingest undercooked pork or untreated water containing the larvae. Once it gets into the human body, it attaches to the intestinal mucosa and grows to full length. The adult tape worm releases cysticerci which can disseminate to different parts of the body. Cysticerci infection of tissues of the central nervous system causes $\mathrm{NCC}^{11-}$ 12.

Cysticidal therapy with albendazole or praziquantel has shown to be effective in reducing the number of cystic lesion in NCC. There has been documentation of neurological exacerbation during the second to fifth day of therapy. For this reason, it is recommended to co-administer praziquantel or albendazole with a steroid to decrease inflammation $^{13}$. The present guidelines by the American Academy of Neurology have suggested that treatment will be done by albendazole and either with dexamethasone or prednisone ${ }^{10}$. Reoccurrence of seizure symptoms is related to the persistence of active brain lesion. Once the NCC lesion clears, the risk of reoccurrence is low ${ }^{14}$.

There are currently no medications recommended for the prevention of NCC traveler who wishes to travel to areas where the NCC prevalence is high. The most effective way to reduce transmission includes, community education on routes of transmission, good personal hygiene such as hand washing to food preparation. Reduction of the potential reservoir can be performed by preventing the transmission of cysticeral infections to pigs ${ }^{15-16}$. NCC is a common disease in developing countries. It has been estimated that 50 million people worldwide have cysticercosis infection. There are many endemic regions includes Central and South America, sub-Saharan Africa, India, and Asia.

The prevalence of cysticercosis varies in different regions. It is often found higher in rural areas, especially where pigs are raised in suboptimal sanitary conditions. Through immigration and international travel, there have been reports of cases this country. It is therefore important for physicians to be aware of the various presentations of the disease and how to diagnose and treat the symptoms promptly. Early detection is very important for a better prognosis. The most effective way to eradicate cysticercal infection will require implementation of strategies to reduce transmission at several stages, such as preventing human tape worm infection due to pork consumption, 
improving sanitary conditions to prevent transmission of cysticercal infections from humans to pigs and measures to interrupt transmission of eggs between humans.

\section{Conclusion}

Neurocysticercosis is a preventable and curable disease to a great extent. A significant level of suspicion can help many patients get relieved of various symptoms including epilepsy through administration of appropriate drugs. Physicians working in disease prone areas should be more alert to run routine antihelminthic programme and engage the people in dietary education.

\section{References}

1. WHO. Seven neglected endemic zoonoses - some basic facts [Online]. Available at: http://www.who.int/zoonoses/neglected_zoonotic_disease s/en/. [Accessed on 22 ${ }^{\text {nd }}$ January, 2017]

2. WHO. Taeniasis/Cysticercosis [Online]. Available at: http://www. who.int/mediacentre/factsheets/fs376/en/. [Accessed on 22 $2^{\text {nd }}$ January, 2017]

3. Del Brutto OH. Neurocysticercosis. Neurohospitalist 2014; 4(4): 205-212

4. Fogang YF, Savadogo AA, Camara M, Toffa DH, Basse $\mathrm{A}$, Sow $\mathrm{AD}$, et al. Managing neurocysticercosis: challenges and solutions. Int J Gen Med 2015; 8: 333-344

5. Goodman KA, Ballagh SA, Carpio A. Case-control study of seropositivity for cysticercosis in Cuenca, Ecuador. Am J Trop Med Hyg 1999; 60(1): 70-74

6. Fleury A, Trejo A, Cisneros H, García-Navarrete R, Villalobos N, Hernandez $M$, et al. Taeniasolium: development of an experimental model of porcine neurocysticercosis. PLoS Negl Trop Dis 2015; 9(8): e0003980

7. Roman G, Sotelo J, Brutto OD, Flisser A, Dumas M, Wadia $\mathrm{N}$, et al. A proposal to declare neurocysticercosis an international reportable disease. Bull World Health Organ 2000;78(3): 399-406

8. CDC. Parasites - cysticercosis [Online]. Available at: http://www.

cdc.gov/parasites/cysticercosis/health_professionals/index. html. [Accessed on 22 ${ }^{\text {nd }}$ January, 2017].

9. Prasad A, Gupta RK, Nath K, Pradhan S, Tripathi M, Pandey C, Mand Prasad KN. What riggers seizures in neurocysticercosis?- A MRI based Study in Pig Farming Community from a District of North India. Parasitol Int 2008;55; 166-171

10. UpToDate. Epidemiology, transmission and prevention of cysticercosis [Online]. Available at: http://www.uptodate.com/contents/ epidemiologytransmission-and-prevention-of-cysticercosis. [Accessed on $22^{\text {nd }}$ January, 2017]

11. Sorvillo FJ, Christopher DeGiorgio C, Waterman SH. Deaths from cysticercosis, United States. Emerg Infect Dis 2007; 13(2): 230- 235

12. Mahanty S, Orrego MA, Mayta H, Marzal M, Cangalaya C, Paredes A, et al. Post-treatment vascular leakage and inflammatory responses around brain cysts in porcine neurocysticercosis. PLoS Negl Trop Dis 2015; 9(3): e0003577

13. Singhi P. Neurocysticercosis. Ther Adv Neurol Disord 2011; 4(2): 67-81

14. Carpio A, Hauser WA. Prognosis for seizure recurrence in patients with newly diagnosed neurocysticercosis. Neurology 2002; 59(11): 1730-1734

15. García HH, Gonz'alez AE, Del Brutto OH, Tsang VCW, Lianos-Zavalaga F, Gonzalvez Guillermo, et al. Strategies for the elimination of taeniasis/cysticercosis. J Neurol Sci 2007; 262(1-2): 153-157

16. Wandra T, Swastika K, Dharmawan NS, Purba IE, Sudarmaja IM, Yoshida T, et al. The present situation and towards the prevention and control of neurocysticercosis on the tropical island, Bali, Indonesia. Parasit Vectors 2015; 8: 148 